\title{
Forecasting the Trend of Traffic Accident Mortality in West Iran
}

\author{
Farzaneh Zolala, ${ }^{1}$ Ali Akbar Haghdoost, ${ }^{1}$ Touraj Ahmadijouybari, ${ }^{2}$ Arash Salari, ${ }^{3}$ Abbas Bahrampour, ${ }^{1}$ \\ Mohamad Reza Baneshi, ${ }^{1}$ and Alireza Razzaghi ${ }^{4,}{ }^{*}$ \\ ${ }^{1}$ Social Determinants of Health Reseach Center, Institute for Futures Studies in Health, Kerman University of Medical Sciences, Kerman, IR Iran \\ ${ }^{2}$ Khomeini Hospital, Kermanshah University of Medical Sciences, Kermanshah, IR Iran \\ ${ }^{3}$ Forensic Medicine in Kermanshah, Kermanshah, IR Iran \\ ${ }^{4}$ Guilan Road Trauma Research Center, Guilan University of Medical Sciences, Rasht, IR Iran \\ "Corresponding author: Alireza Razzaghi, Guilan Road Trauma Research Center, Guilan University of Medical Sciences, Rasht, IR Iran. Tel: +98-9354745949, Fax: +98-33338373, \\ E-mail: alirezarazzaghi_21@yahoo.com
}

Received 2015 July 07; Revised 2015 December 24; Accepted 2016 January 02.

\begin{abstract}
Background: Traffic accidents are the main cause of deaths in developing countries. Fatalities due to traffic accidents are assessed through a three-year time series forecast.

Objectives: The aim of this study is to use trend assessment to predict traffic accident fatalities for January 2013 to December 2015 in Kermanshah province, Iran.

Materials and Methods: This is a historical longitudinal study using time series analysis to identify the best fit model. The criteria of MSE (mean square of error) were used to determine the model with the best goodness of fit. The model that had the smaller MSE value was introduced as a suitable model. The selected model was used to forecast the number of deaths related to traffic accidents in the next three years.

Results: A decreasing trend was observed in accident mortality. The highest and lowest deaths were seen annually in the spring and autumn months, respectively. The SARIMA $(0,0,0) \times(1,1,1) 12$ model was identified as the best-fit model for data. Prediction values of traffic accident fatalities showed a decreasing trend in deaths in the coming years.

Conclusions: Applying this information can be useful to policy makers and managers for planning and implementing special interventions to prevent and limit future accidental deaths.
\end{abstract}

Keywords: Traffic Accidents, Mortality, Forecasting

\section{Background}

The high rate of Road Traffic Injuries (RTIS) poses a growing public health problem throughout the world (1). Based on a world health organization (WHO) report, by 2020 road traffic injuries will be the third leading cause of illness and injury in the world (2). Implementing effective interventions in high-income countries has mitigated and decreased traffic accidents and injury problems. However, this problem requires special attention in lowand middle-income countries (3). Traffic deaths and injuries will increase by as much as $80 \%$ between 2000 and 2020 , based on forecasting information implemented in low- and middle-income countries (1).

In Iran specifically, road traffic accidents and fatalities are increasing at an alarming rate (4). Based on estimates, Iranian road traffic accidents lead to about 30,000 deaths annually (5). This problem has become the main priority for public health policy, and several interventions have been implemented to enhance prevention and con- trol (6). Appropriate planning for these interventions requires methods that effectively assess health events and indicate possible outcomes in future years (7). Using trend assessment of the data and forecasting methods can improve communication between scientists and policy makers, and ultimately lead to better planning and decision making (7). Having predictive information about accident mortality can provide important information on upcoming changes in accident trends. There are different statistical methods to forecast future conditions. This study uses time series analysis; its main purpose is modeling and forecasting (8). Modeling and forecasting traffic fatalities can provide insight for policy-makers to help them adjust their policies and implement effective countermeasures (8).

\section{Objectives}

Kermanshah is one of the provinces in west Iran. Like other provinces in Iran, Kermanshah suffers from traffic ac- 
cidents and the accompanying fatalities and injuries. However, there is a lack of related studies about trend assessment and forecasting. Thus, the aim of this study is to evaluate trend assessment and forecast the traffic fatalities from January 2013 to December 2015 in Kermanshah province, Iran.

\section{Materials and Methods}

Through a historical analytical study, fatal crash data have been obtained from Kermanshah forensic experts for January 2005 to December 2012. Kermanshah province is located in the middle of western Iran. Based on the 2011 census, the population of Kermanshah province is $1,945,227$. The months of April, May and June are the spring months; July, August and September are summer; October, November and December are autumn; and January, February and March are the winter months.

A time series analysis, including Box-Jenkins models, was used for trend assessment and selecting a best fit model, so as to forecast accident mortality trends in the next three years. Time series analysis is an endogenous statistical method. This means that the method is based on a historic pattern of actual observations. The identified pattern will be applied to forecast events in the future. Time series modeling is accomplished through three steps: (1) identification, (2) estimation, and (3) diagnosis. These three steps may be repeated several times to achieve the best fit model.

Before performing time series analysis, the quality of the collected data was assessed in terms of data integrity. Based on data integrity, a monthly period was chosen. Time series modeling is meaningful when the stability of a time sequence is established in terms of mean and variance. It is necessary for a stationary mean and variance to be established. Moreover, when data have seasonal effects, it is important to control this effect. Therefore, before the forecasting procedure, the stability of the observation was examined by plotting the trend. Box-Cox transformation was applied to remove non-stationary variance. In order to remove the seasonality variation and trend from the observed time series, seasonal differencing and order differencing, respectively, were applied to the data. The patterns of the autocorrelation function (ACF) and the partial autocorrelation function (PACF) plots are used to identify potential models. In this study, several different models were identified through analysis of the ACF and PACF plots, including AR (auto regressive), MA (moving average), ARMA (auto regressive moving average), ARIMA (autoregressive moving integrated moving average), and SARIMA (seasonality autoregressive moving integrated moving average).
After diagnosing the best fit model, the identified models were compared by least mean square error (MSE) and ACF and PACF residual plots as goodness of fit criteria. The lowest value of the MSE represents the goodness of fit among the identified models. The analysis of model residuals was based on the ACF and PACF residual plots. In order to select the best fit model by residual analysis, the ACF and PACF residual plots should not be significantly different from zero. In other words, no spike should be observed from the boundary lines around the ACF and PACF functions with $95 \%$ confidence intervals. The correlation coefficient was used to examine the quality of each selected model in comparison with the actual data. After examining different models, the SARIMA model was used for forecasting. The SARIMA model is an extended model of ARIMA, which consists of auto-regression, difference, and moving average: (p, d, q) (P, D, Q). The parameters of p, d, and q represent the auto-regressive, integrated, and moving average model in the non-seasonal part. The parameters of $\mathrm{P}, \mathrm{D}$, and $\mathrm{Q}$ represent the auto-regressive, integrated and moving average model in the seasonal part. The diagnosed best fit model was used to predict traffic fatalities in Kermanshah between January 2013 and December 2015. Analyses were performed using Minitab 17 software $(9,10)$.

\section{Results}

The total number of traffic fatalities in Kermanshah from January 2005 to December 2012 was 4980. These fatalities included pedestrians and motorcyclists as well as automobile drivers and passengers. The highest and lowest fatalities, respectively, were observed in April (mean = $70.13, \mathrm{SD}=9.26)$ and November $($ mean $=31.25, \mathrm{SD}=9.63)$, annually.

Plotting the deaths trend during the studied years showed an additive trend, including a seasonality and an order trend. In other words, there is a decreasing trend in the accidents' mortality and a seasonality pattern according to decomposition with lag 12 (Figure 1). This means that there is non-stationary variance and mean. A Box-Cox transformation, with seasonality differencing with lag 12 , was used to remove the non-stationary conditions in variance and seasonality variation, respectively. The time series reached a stationary condition without requiring an application of the order difference. After removing nonstationary conditions, autocorrelation function (ACF) and partial ACF (PACF) plots were plotted to identify the type of ARIMA and SARIMA model (Figure 2).

Based on ACF and PACF plots, parameters for d and D equaled 0 and 1 , respectively. The level of $\mathrm{p}$ and $\mathrm{P}$ was 0 and 1 , respectively, as were $\mathrm{q}$ and $\mathrm{Q}$. The suggested models, 


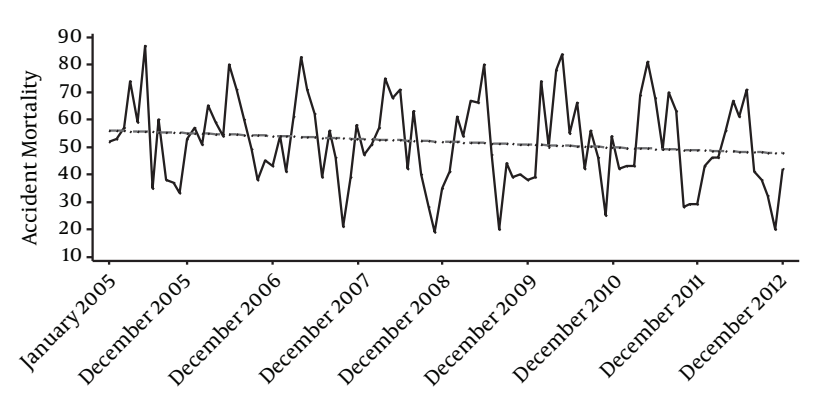

Figure 1. Trend Assessment of Accident Mortality in Kermanshah From 2005 until 2012

as well as the MSE values and other diagnostic parameters that could be fitted to the data, are shown in (Table 1 ).

The SARIMA $(0,0,0)(1,1,1) 12$ model had the lowest MSE value of $118.95(\mathrm{df}=81)$. Also, the ACF and PACF residual plots, i.e., the difference between the model and the actual series, are not significantly different from zero. This means that the model is the best fit model and applicable for forecasting. The Pearson correlation coefficient of the model was $0.75(\mathrm{P}=0)$. The resulting model was used to forecast traffic fatalities between January 2013 and December 2015 with $95 \%$ confidence. The forecasting plots of traffic fatalities are presented in (Figure 3). According to the forecasting values for the three years, there were significantly more traffic fatalities in spring, with a mean and standard deviation of 65.961 .22 , more than other seasons $(F=160.5, P=0)$. Moreover, there is a decreasing trend in the occurrence of traffic accidents and deaths.

\section{Discussion}

Results show a decreasing trend in traffic fatalities during the study period (January 2005 to December 2012) in Kermanshah province. However, slight variations exist in death rates during those years. Overall, the declining trend of traffic fatalities is in spite of increasing population and vehicles during the study period. These traffic fatalities were expected due to national interventions which have been implemented to abate traffic accidents in recent years. Various policies and activities which have been implemented to reduce traffic accidents and their fatalities include traffic regulation reform, new legislation such as mandatory helmet usage for motorcyclists and seat belts for car drivers, increasing traffic violation penalties for drivers and motorcyclists, and improving traffic control device warrants. Based on previous studies, deaths related to road traffic injuries have decreased from 2007. This reduction of the death rate in 2007 compared to previous years could likewise be attributed to certain interventions, such as traffic regulations and new driving policies which started in 2005 (11).

The results of the trend assessment in this study is consistent with a similar trend study which had been done in all provinces of Iran, using the police records of road traffic deaths, from January 2005 to December 2012 (11). The SARIMA $(0,0,0)(1,1,1) 12$ model was selected as the best fit model and applied for predicting the traffic fatalities. SARIMA is an expanded version of the ARIMA model which is influenced by seasonality factors. According to the results, a high rate of traffic fatalities occurred in the spring season months. The high number of trips in spring affects the level of accidents and their related fatalities. Forecasting the deaths due to traffic accidents in the next threeyear period revealed a similar seasonal pattern. This highlights the need for police and policy makers to apply suitable interventions to reduce traffic crashes in months with high numbers of fatalities, i.e., the spring months. That could mean employing more personnel as traffic police to enforce traffic laws and improving the emergency medical rescue system to save more people who are injured in traffic accidents. Based on previous research, decreasing the emergency medical services' response time significantly decreases fatalities in accidents (12).

Moreover, it is interesting to note the distribution of traffic fatalities among vehicle drivers, motorcyclists, passengers, and pedestrians. Several human factors can affect the occurrence of accidents and injuries (13). For example, pedestrians are at high risk of being involved in traffic accidents because of their vulnerability. Pedestrians are involved in many road traffic injuries and a considerable percent of traffic deaths occur among them $(14,15)$. Therefore, more attention and consideration is required by policy makers, including educating road users on traffic regulations.

Based on this forecasting pattern, there will be a decreasing trend of traffic fatalities in the future. This finding is in agreement with another recent study (16). This decreasing trend is due to multiple interventions which have been implemented in recent years by policy makers in Iran. Unfortunately, one of the main limitations of this study is a lack of precise information about the various interventions which have been implemented to prevent and control traffic accidents in previous years. Using these interventions as an independent variable could enable us to assess the effect of each intervention in the trend of fatal traffic accidents.

\subsection{Conclusion}

The prediction values of deaths related to traffic accidents show that there will be a decreasing trend in deaths 
A

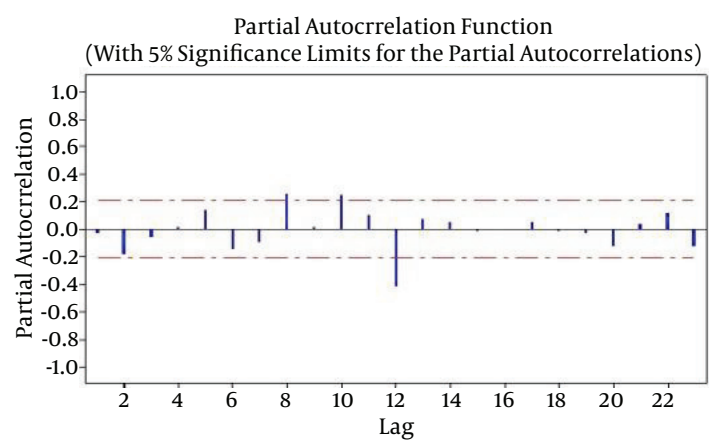

B

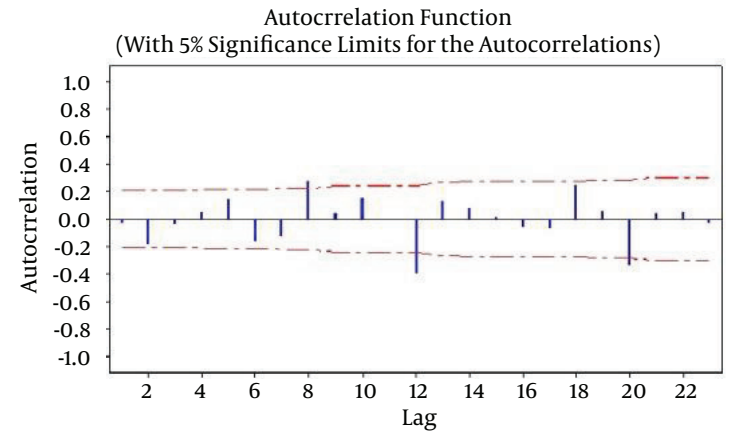

Figure 2. Partial Autocorrelation and Autocorrelation Function Plots of Accident Mortality in Kermanshah, After Removing the Non-Stationary Data

Table 1. Tests to Compare the Adequacy of the Identified Model

\begin{tabular}{|c|c|c|c|c|c|c|c|c|c|c|}
\hline \multirow[t]{2}{*}{ Model } & \multicolumn{3}{|c|}{ T-Test } & \multicolumn{2}{|c|}{ Residual Plots } & \multicolumn{2}{|c|}{ Ljung-Box (Lag12) } & \multicolumn{2}{|c|}{ Correlation } & \multirow[t]{2}{*}{ MSE, DF } \\
\hline & Lag & t & PValue & ACF & PACF & Chi-Square & P Value & $\mathbf{r}$ & PValue & \\
\hline $\operatorname{SARIMA}(\mathbf{0}, \mathbf{0}, \mathbf{0})(\mathbf{1}, \mathbf{1}, \mathbf{1}) 12$ & & & & Not significant & Not significant & 15.9 & 0.07 & 0.75 & 0.000 & $118.95,81$ \\
\hline SAR & 12 & 0.98 & 0.03 & & & & & & & \\
\hline SMA & 12 & 6.67 & 0.000 & & & & & & & \\
\hline $\operatorname{SARIMA}(\mathbf{0}, \mathbf{0}, \mathbf{0})(\mathbf{0}, \mathbf{1}, \mathbf{1}) 12$ & & & & Not significant & Not significant & 15.4 & 0.11 & 0.75 & 0.000 & $119.84,82$ \\
\hline SMA & 12 & 8.36 & 0.000 & & & & & & & \\
\hline $\operatorname{SARIMA}(\mathbf{0}, \mathbf{0}, \mathbf{0})(\mathbf{1}, \mathbf{1}, \mathbf{0}) 12$ & & & & Not significant & Not significant & 17.1 & 0.07 & 0.63 & 0.001 & $163.2,82$ \\
\hline SAR & 12 & 5.45 & 0.000 & & & & & & & \\
\hline
\end{tabular}

Abbreviations: ACF, autocorrelation function; PACF, partial autocorrelation function; AR, autoregressive; MA, moving average; SARIMA, seasonal autoregressive integrated moving average; MSE; mean square of errors.

${ }^{\mathrm{a}}$ Coefficient Model and Actual Data.

${ }^{\mathrm{b}}$ Parameters Equalization Values With Zero.

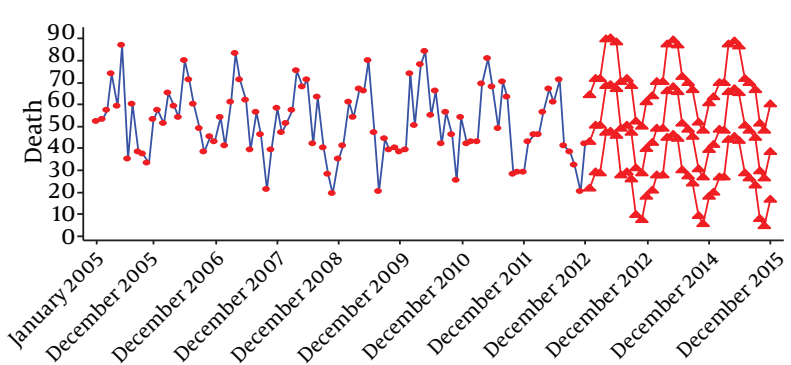

Time (Month)

Figure 3. Forecasting Plot of Accident Mortality From January 2013 to December 2015. Time Series Plot for Death (With Forecasts and Their 95\% Confidence Unit)

in coming years. The interventions which have been applied in recent years have been useful. Also, the highest number of traffic fatalities will occur in spring season. Policy makers and managers should pay more attention to prevent road-related accidents in the springs of future years.

\section{Acknowledgments}

The authors wish to thank the research center for modeling in health, institute for futures in health, Kerman University of Medical Sciences. Also, we would like to thank Mr. Mehdi Moradi who provided us with data.

\section{References}

1. Peden M, Scurfield R, Sleet D, Mohan D, Hyder AA, Jarawan E. World report on road traffic injury prevention Geneva: World Health Organization; 2004.

2. Naci H, Chisholm D, Baker TD. Distribution of road traffic deaths by road user group: a global comparison. Inj Prev. 2009;15(1):55-9. doi: 10.1136/ip.2008.018721. [PubMed: 19190278].

3. Bishai DM, Hyder AA. Modeling the cost effectiveness of injury interventions in lower and middle income countries: opportunities and challenges. Cost Eff Resour Alloc. 2006;4(2).

4. Naghavi M, Shahraz S, Bhalla K, Jafari N, Pourmalek F, Bartels D, et al. Adverse health outcomes of road traffic injuries in Iran after rapid motorization. Arch Iran Med. 2009;12(3):284-94. [PubMed: 19400607].

5. Soori H, Royanian M, Zali AR, Movahedinejad A. Road traffic injuries in Iran: the role of interventions implemented by traffic police. Traffic Inj Prev. 2009;10(4):375-8. doi: 10.1080/15389580902972579. [PubMed: 19593716]. 
6. Razzaghi A, Bahrampour A, Baneshi MR, Zolala F. Assessment of trend and seasonality in road accident data: an Iranian case study. Int J Health Policy Manag. 2013;1(1):51-5. doi: 10.15171/ijhpm.2013.08. [PubMed: 24596836].

7. Lemos MC, Finan TJ, Fox RW, Nelson DR, Tucker J. The use of seasonal climate forecasting in policymaking: lessons from Northeast Brazil. Climatic Change. 2002;55(4):479-507. doi:10.1023/A:1020785826029.

8. Commandeur JJ, Bijleveld FD, Bergel-Hayat R, Antoniou C, Yannis G, Papadimitriou E. On statistical inference in time series analysis of the evolution of road safety. Accid Anal Prev. 2013;60:424-34. doi: 10.1016/j.aap.2012.11.006. [PubMed: 23260716].

9. Yannis G, Antoniou C, Papadimitriou E. Autoregressive nonlinear time-series modeling of traffic fatalities in Europe. Euro Trans Res Rev. 2011;3(3):113-27. doi: 10.1007/s12544-011-0055-4.

10. Minitab . 2015. Available from: http://www.minitab.com/en-us.

11. Bahadorimonfared A, Soori H, Mehrabi Y, Delpisheh A, Esmaili A, Salehi M, et al. Trends of fatal road traffic injuries in Iran (20042011). PLoS One. 2013;8(5):e65198. doi: 10.1371/journal.pone.0065198. [PubMed: 23724132].
12. Gonzalez RP, Cummings GR, Phelan HA, Mulekar MS, Rodning CB. Does increased emergency medical services prehospital time affect patient mortality in rural motor vehicle crashes? A statewide analysis. Am J Surg. 2009;197(1):30-4. doi: 10.1016/j.amjsurg.2007.11.018. [PubMed: 18558397].

13. Bungum TJ, Day C, Henry LJ. The association of distraction and caution displayed by pedestrians at a lighted crosswalk. J Community Health. 2005;30(4):269-79. [PubMed: 15989209].

14. Nantulya VM, Reich MR. The neglected epidemic: road traffic injuries in developing countries. BMJ. 2002;324(7346):1139-41. [PubMed: 12003888].

15. Hatfield J, Murphy S. The effects of mobile phone use on pedestrian crossing behaviour at signalized and unsignalized intersections. Accid Anal Prev. 2007;39(1):197-205. doi: 10.1016/j.aap.2006.07.001. [PubMed: 16919588].

16. Bahadorimonfared A, Soori H, Mehrabi Y, Roodsari M, Esmaeili A, Salehi M. Prediction of accident's deaths in Iran. Res Medicine. 2012;36(1):7-11. 